\title{
Diacronie
}

Studi di Storia Contemporanea

$N^{\circ} 30,2$ | 2017

Ponti fra nazioni e continenti

\section{Renato Camurri (a cura di), Pensare la libertà. I quaderni di Antonio Giuriolo}

\section{Camilla Poesio}

\section{Q OpenEdition \\ 1 Journals}

\section{Edizione digitale}

URL: http://journals.openedition.org/diacronie/5902

DOI: 10.4000/diacronie.5902

ISSN: 2038-0925

Editore

Association culturelle Diacronie

Notizia bibliografica digitale

Camilla Poesio, «Renato Camurri (a cura di), Pensare la libertà. I quaderni di Antonio Giuriolo », Diacronie [Online], N 30, 2 | 2017, documento 8, Messo online il 29 juillet 2017, consultato il 25 septembre 2020. URL : http://journals.openedition.org/diacronie/5902 ; DOI : https://doi.org/10.4000/diacronie.5902 


\title{
Diacronie
}

Studi di Storia Contemporanea

30, 2/2017

Ponti fra nazioni e continenti: diplomazia, immaginari e conoscenze tecniche

\section{RECENSIONE: Renato CAMURRI (a cura di), Pensare la libertà. I quaderni di Antonio Giuriolo, Venezia, Marsilio, 2016, 507 pp.}

\author{
A cura di Camilla POESIO
}

Per citare questo articolo:

POESIO, Camilla, «RECENSIONE: Renato CAMURRI (a cura di), Pensare la libertà. I quaderni di Antonio Giuriolo, Venezia, Marsilio, 2016, 507 pp.», Diacronie. Studi di Storia Contemporanea : Ponti fra nazioni e continenti: diplomazia, immaginari e conoscenze tecniche, 30, 2/2017, 29/7/2017,

URL: < http://www.studistorici.com/2017/07/29/poesio_numero_30/ >

Diacronie Studi di Storia Contemporanea $\rightarrow$ http://www.diacronie.it

Rivista storica online. Uscita trimestrale.

redazione.diacronie@hotmail.it

Comitato di direzione: Naor Ben-Yehoyada - João Fábio Bertonha - Christopher Denis-Delacour - Maximiliano Fuentes Codera Anders Granås Kjøstvedt - John Paul Newman - Deborah Paci - Niccolò Pianciola - Spyridon Ploumidis - Wilko Graf Von Hardenberg

Comitato di redazione: Jacopo Bassi - Luca Bufarale - Gianluca Canè - Fausto Pietrancosta - Alessandro Salvador - Matteo Tomasoni Diritti: gli articoli di Diacronie. Studi di Storia Contemporanea sono pubblicati sotto licenza Creative Commons 3.0. Possono essere

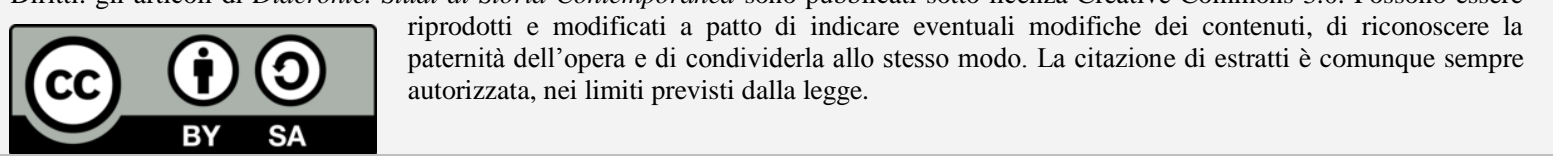




\title{
8/ RECENSIONE: Renato CAMURRI (a cura di), Pensare la libertà. I quaderni di Antonio Giuriolo, Venezia, Marsilio, 2016, 507 pp.
}

\author{
A cura di Camilla POESIO
}

Non è facile fare la recensione di questo volume perché, nel momento in cui ci si appresta ad affrontare le quasi cinquecento pagine che lo compongono, ci si trova davanti, di fatto, non ad uno ma a due libri. Il primo è il bel saggio introduttivo del curatore del volume, Renato Camurri, professore di storia dell'Europa contemporanea all'Università di Verona e autore di numerose ricerche sulla storia delle classi dirigenti e delle élites nell'età liberale ${ }^{1}$, ma anche sull'emigrazione, sull'esilio e sugli intellettuali²; il secondo libro sono i quaderni che Antonio Giuriolo scrisse su temi disparati, dalla filosofia alla letteratura, dalla storia alla politica, e che ci illustrano la significativa cifra culturale che caratterizzò l'intellettuale veneto. L'obiettivo è quello di capire il suo mondo e le sue letture per delineare una figura di spicco dell'antifascismo italiano, vicina a Norberto Bobbio, Carlo Ludovico Ragghianti, Aldo Capitini - per dirne alcuni - e che è rimasta in ombra per troppi anni.

Antonio Giuriolo (San Pietro a Castello di Arzignano, Vicenza - 12 febbraio 1912, Corona di Lizzano in Belvedere, Bologna, 12 dicembre 1944), figlio dell'avvocato Pietro, antifascista laico e socialista militante tanto da subire le violenze degli squadristi, e di Marina Arrenghini, donna profondamente cattolica, secondogenito di tre fratelli, frequentò il ginnasio prima a Bologna nel collegio San Luigi e poi nel liceo Pigafetta di Vicenza, dove la famiglia si trasferì nell'autunno del 1922. Nel 1930 si iscrisse alla Facoltà di Lettere dell'Università di Padova e si laureò con una tesi sulla poesia di Antonio Fogazzaro.

I quaderni - solo in parte pubblicati in questo volume - sono stati donati all'Istituto Storico della Resistenza e dell'Età Contemporanea della provincia di Vicenza "Ettore Gallo" dagli eredi di

\footnotetext{
${ }^{1}$ Per citarne alcuni: CAMURRI, Renato, ZURITA, Rafael, Les élites en Italia y en Espana, 1850-1922, Valencia, Puv, 2008; CAMURRI, Renato, «Introduzione», in Ricerche di Storia Politica : Notabili e sistemi notabilari nell'Europa liberale, XV, 3/2012, pp. 257-260; ID., «I Tutori della nazione: i "grandi notabili" e l'organizzazione della politica nell'Italia liberale», ibidem, pp. 261-278.

${ }^{2}$ CAMURRI, Renato (a cura di), Franco Modigliani. L'Italia vista dall'America. Riflessioni e battaglie di un esule, Torino, Bollati Boringhieri, 2010; ID. (a cura di), Max Ascoli. Antifascista, intellettuale, giornalista, Milano, Franco Angeli, 2012; ID. (a cura di), Gaetano Salvemini. Lettere Americane. 1927-1949, Roma, Donzelli, 2015; CAMURRI, Renato, GENTILE, Emilio, The March on Rome: how antifascists understood the origins of totalitarianism (and coined the word), Roma, Viella, 2013.
} 
Antonio Giuriolo nel 2004, un lascito che ha permesso di dare vita a un prezioso fondo costituito da lettere, articoli di stampa e quarantasette quaderni dalla copertina nera. Questi ultimi non sono datati: non deve essere stato facile, dunque, ricostruire una loro cronologia. Camurri ha risolto il problema attraverso congetture, verifiche incrociate e con lo strumento della comparazione, confrontando cioè i percorsi di altri coetanei di Giuriolo. Ne ha concluso che una prima parte dei quaderni sia stata scritta tra il 1930 e il 1935, una seconda tra il 1937 e il 1943, inframezzati da un diario redatto negli anni in maniera discontinua (inizia il 5 settembre 1936 e si ferma il 9 settembre, riprende dal 16 maggio 1939 al 19 settembre, si ferma di nuovo per tre anni e riprende nel 1942, senza data ma sicuramente il 19 gennaio, il giorno dopo la morte del padre), anni durante i quali Giuriolo conobbe e frequentò l'antifascismo organizzato che gravitava a Firenze, attorno alla rivista «La Nuova Italia», e a Parigi. Se ricostruire una cronologia di questi quaderni deve essere stato compito non grato, altrettanto deve esserlo stato decifrarli: il particolare di una pagina autografa di Giuriolo, che si vede sulla copertina del volume, ci dà un piccolo assaggio di una scrittura fitta e minuta intervallata da cancellature.

Il libro si divide in due grandi parti. La prima è costituta da un denso saggio del curatore, la seconda da quindici di quarantasette quaderni autografi. Già qualche anno fa Camurri aveva tracciato un profilo biografico e politico di Giuriolo3; adesso lo storico inquadra e spiega la figura dell'intellettuale facendola emergere dai suoi scritti e dalle sue riflessioni su Machiavelli, Tocqueville, Cavour, Guido Calogero, Henri De Man.

Attraverso un difficile lavoro filologico il curatore ha selezionato i quaderni forse più interessanti, quelli in cui Giuriolo affronta concetti di grande portata come libertà, uguaglianza, patria, educazione, Storia, marxismo, socialismo. Lette con attenzione queste riflessioni - talvolta semplici annotazioni su cui Giuriolo si promette di tornare in un secondo momento - non sono altro che la visione politica e umana di un uomo che dello studio fece non un rifugio sterile, bensì un modo per prepararsi alla lotta antifascista. Se la lettura dei classici latini e greci lo aiutarono a sviluppare riflessioni acute sul concetto di libertà e sulla figura del tiranno, Machiavelli gli servì per affrontare il rapporto tra potere e cittadini. Pertanto, come spiega Camurri, «questi commenti non sono meri esercizi di erudizione. Essi vanno [...] sempre letti cercando di contestualizzarli e di decodificare quello che l'autore vuole dissimulare usando un linguaggio in alcuni casi volutamente evocativo»4.

\footnotetext{
${ }^{3}$ CAMURRI, Renato, Tra mito e antimito: note sulla formazione di Antonio Giuriolo, in ID. (a cura di), Antonio Giuriolo e il "partito della democrazia", Verona, Cierre, 2008, pp. 31-52; ID., Il capitano con gli occhi da bambino, cit., pp. 11-15.

${ }^{4}$ CAMURRI, Renato (a cura di), Pensare la libertà. I quaderni di Antonio Giuriolo, Venezia, Marsilio, 2016, p. 133.
} 
A ciò mi permetterei di aggiungere che, non di rado, alcune affermazioni di Giuriolo sono vere e proprie prese di posizione politiche critiche al regime fascista. Per fare alcuni esempi, scrive Giuriolo:

Come si vede chiaramente, lo Stato tranquillo e ordinato, senza dissenso e senza lotte interne, non è l'ideale del M. [Machiavelli], il quale vedeva anzi in quei dissensi lo stimolo più potente a un rinforzamento della libertà e della grandezza 5 .

Oppure:

La potenza di uno stato non si misura dalla permanenza più o meno lunga di un governo (v. la decadenza di Venezia) ma dalla sua capacità di rinnovarsi, di adattarsi, di trasformarsi secondo le contingenze $e^{6}$.

È significativa una frase del genere se contestualizzata a un presente in cui il regime fascista si proponeva, invece, come unico scopo quello di durare. E ancora:

L'ideale del principe $\mathrm{m}$. [machiavelliano] non è quello che fonda il suo dominio su un programma di violenza e di costrizione, ma quello che si fa amare dai sudditi, che rispetta le leggi ${ }^{7}$.

Fino ad arrivare:

Scopo essenziale della politica non è dare effimero lustro a un popolo e a un individuo, ma prepararlo per le lotte del futuro, educarlo con istituzioni durature [...]

Per questo l'opera dei dittatori è spesso effimera: non si curano del futuro, non si negano la propria personalità nelle istituzioni: il loro successo dipende infine da "un colpo di pistola"».

Per utilizzare una categoria gobettiana Giuriolo fu un esule in patria - così titola uno dei capitoli del volume - perché effettivamente la sua scelta fu quella di un esilio spirituale, esistenziale, di una estraneità profonda e di un ritiro nello studio dei classici, della filosofia, della scienza politica e della letteratura non per fuggire dal presente, bensì per imparare, prendere ispirazione, "imitare", (proprio come aveva fatto a sua volta Machiavelli da lui tanto studiato) dal passato e agire sul presente in vista della costruzione di un'altra patria una volta sconfitta la dittatura.

\footnotetext{
${ }^{5}$ Ibidem, p. 317.

${ }^{6}$ Ibidem, p. 315.

${ }^{7}$ Ibidem, p. 345

${ }^{8}$ Ibidem, p. 323.
} 
Parlando forse a sé stesso, riteneva coraggiosa l'attività culturale antifascista al pari di colui che agisce:

Il coraggio non appartiene solo al martire o all'eroe, ma anche al filosofo, al poeta, i quali devono affrontare lotte interiori durissime [...] qualunque forma d'attività impegna tutta la personalità; moralmente nemmeno, perché ogni fede impone all'individuo il sacrificio totale, quando sia necessario (v. Bruno) $)^{9}$

Ma Giuriolo non si limitò a questo. Nel 1937 cominciò a frequentare ambienti in cui giravano copie clandestine di Socialismo liberale di Carlo Rosselli, libro vietato a cui l'intellettuale arrivò grazie a Carlo Ludovico Ragghianti; lesse Elementi di un'esperienza religiosa di Aldo Capitini uscito in quell'anno e il Primo manifesto del liberalsocialismo di Guido Calogero divulgato nel corso della primavera del $1940^{10}$. Conobbe, inoltre, Luigi Russo, Francesco Flora, Aldo Capitini, i giovani vicini alla rivista «La Nuova Italia», tra cui Enriques Agnoletti. Nel 1938, ospite della zia materna Anita Arreghini Hugonin, si recò a Parigi dove frequentò l'antifascismo internazionale, ma soprattutto fece vita politica attiva incontrando militanti antifascisti, operai, emigranti italiani, sindacalisti. Infine, subito dopo l'8 settembre, entrò nella Resistenza dove trovò la morte in combattimento.

È in questa ottica, allora, che vanno letti i suoi ragionamenti, che partono da letture antiche, su grandi questioni come la libertà, l'uguaglianza, le strutture economiche, la forma di stato - se centralizzato o decentrato. Attraverso gli appunti e le sue riflessioni, capiamo che è a Rosselli che si sentì più vicino politicamente: Giuriolo si può cioè collocare politicamente nel filone del socialismo liberale - né il liberalismo, né la democrazia, né il comunismo, né il nazionalfascismo erano esperienze politiche da tenere a modello ${ }^{11}-$, sia per la nozione di libertà, secondo la quale non si è liberi per nascita ma per comportamento e attività di vita e di coscienza, sia per quella di dignità (che non è condizione innata o causale ma che si acquisisce attraverso le proprie azioni), sia per questioni relative al decentramento e alla valorizzazione delle autonomie locali, temi ampiamente discussi nell'ambiente gravitante attorno a Giustizia e Libertà. Scrive il curatore:

Di lui direi che è stato un giovane con una forte vocazione al lavoro intellettuale; profondamente ancorato ad una precisa tradizione culturale italiana ma dotato di una mentalità aperta, cosmopolita [...]

\footnotetext{
${ }^{9}$ Ibidem, p. 452.

${ }^{10}$ ROSSELLI, Carlo, Socialismo liberale, s.l., Edizioni di Giustizia e Libertà, [1944]; CAPITINI, Aldo, Elementi di un'esperienza religiosa, Bari, Laterza, 1937; CALOGERO, Guido, Primo manifesto del liberalsocialismo, 1940, Roma, s.e., 1945.

${ }^{11}$ CAMURRI, Renato (a cura di), Pensare la libertà, cit., p. 212.
} 
La sua collocazione politica è nel filone del socialismo liberale di matrice rosselliana, con forti venature libertarie e antitotalitarie ${ }^{12}$.

Intimamente convinto dell'importanza nella società del ruolo dell'educazione come elemento fondamentale per la formazione politica e morale del popolo, la cultura, lo studio, i libri servirono a Giuriolo per rafforzare un senso profondo di cittadinanza democratica e di partecipazione politica e dare vita a un nuovo umanesimo, a una nuova società. Sono indicative in questo senso le sue riflessioni sulla storia:

Intendo per storia tutto quello che gli uomini hanno compiuto [...] a me importa intendere cosa [i personaggi storici] compirono; è la loro opera insomma che non appartiene più a loro come individui, ma appartiene alla storia, nella quale vive. La storia degli individui ha perciò solo un valore in quanto essi vengono legati all'umanità, per la quale operano e in riferimento alla quale soltanto ha senso la loro azione13.

Dotato di un grande carisma, diventò maestro de I piccoli maestri, il gruppo di studenti universitari di cui faceva parte anche Luigi Meneghello ${ }^{14}$, più giovani di lui di una decina di anni essendo nati intorno agli inizi degli anni Venti, che salirono sull'Altopiano di Asiago per combattere i nazifascisti. «Finalmente avevo trovato ciò che inconsciamente cercavo: un maestro di pensiero e d'azione» ${ }^{15}$, scrisse uno di loro.

$\mathrm{Fu}$ un intellettuale che dal pensiero passò all'azione: nel 1936 rifiutò la tessera del Partito nazionale fascista, decisione che lo interdisse dall'insegnamento delle scuole pubbliche e che lo costrinse a dare lezioni private e per questo è stato definito professore senza cattedra; nell'estate del $1942 \mathrm{fu}$ fondatore del Partito d'Azione nel Vicentino e nel 1944, il 12 dicembre, a trentadue anni, morì in combattimento alla guida di una delle due formazioni della Brigata Matteotti a La Corona, nei pressi di Lizzano Belvedere sull'Appenino tosco-emiliano.

Oltre all'evidente utilità pratica di aver riportato fedelmente alcuni scritti, finora accessibili solo in archivio, questo libro ha un altro grande merito: quello di avere valorizzato la figura di questo intellettuale all'interno dell'antifascismo italiano, strappandolo all'oblio in cui era stato relegato e demolendo quel mito-Giuriolo creato dagli scritti di Norberto Bobbio ${ }^{16}$ e dai romanzi di Luigi Meneghello, che ne aveva fatto un eroe per la sua coerenza e rettitudine, per la sua biografia

\footnotetext{
${ }^{12}$ Ibidem, p. 179.

${ }^{13}$ Ibidem, p. 285.

${ }^{14}$ MENEGHELLO, Luigi, I piccoli maestri, Milano, Feltrinelli, 1964.

${ }^{15}$ CAMURRI, Renato (a cura di), Pensare la libertà, cit., p. 29.

${ }^{16}$ BOBBIO, Norberto, L'uomo e il partigiano, in Per Antonio Giuriolo, Vicenza, s.n., 1966, pp.19-32, ripubblicato poi in BOBBIO, Norberto, Italia civile. Ritratti e testimonianze, Firenze, Passigli, 1986, pp. 311-323 e, recentemente, nel Meridiano in ID., Etica e politica. Scritti di un impegno civile, Milano, Mondadori, 2009, pp. 544-558.
} 
esemplare culminata con una morte altrettanto esemplare. La mitizzazione della figura del “capitano Toni" aveva significato anche mitizzare l'esperienza intransigente e contraria a ogni compromesso dell'azionismo. Eppure, nonostante questa sorta di aura sacrale, di Giuriolo non si è più parlato pubblicamente dopo l'intervento di Bobbio del 1948 e quello, sempre del filosofo, del 1964 in occasione dei vent'anni dalla morte dell'intellettuale, «complice anche il ritardo con il quale la storiografia italiana iniziò a occuparsi della storia del Partito d'Azione, ritardo che aveva provocato il "congelamento" del suo profilo intellettuale [...]»"17.

Camurri lo ha riesumato non solo per rendere giustizia all'uomo-Giuriolo, ma anche per aggiungere un tassello in più al variegato mosaico dell'antifascismo italiano:

[L'esperienza di Giuriolo] ci insegna che non basta ricostruire le vicende dei gruppi consolidati organizzati secondo appartenenze che rimandano alle classiche famiglie politiche. Occorre intrecciare queste conoscenze con storie individuali e i percorsi dei piccoli gruppi. [...] È a queste biografie, a queste storie individuali, a questi materiali che bisogna guardare, con rispetto e ammirazione, per capire cosa è veramente stata la Resistenza italiana ${ }^{18}$.

${ }^{17}$ CAMURRI, Renato (a cura di), Pensare la libertà, cit., p. 11.

${ }^{18}$ Ibidem, pp. XIV-XV. 


\section{L'AUTORE}

Camilla POESIO si è addottorata presso l'Università Ca' Foscari di Venezia in cotutela con la Freie Universität Berlin; collabora con l'Università di Paderborn e da gennaio 2017 sarà Visiting Fellow presso il Department of History and Civilization del European University Institute; è Cultore della materia presso il DSLCC-Università Ca' Foscari Venezia. Ha vinto con la sua tesi di dottorato il Premio Nicola Gallerano 2009 e il Premio Ettore Gallo 2009. È autrice dei volumi Reprimere le idee, abusare del potere. La Milizia e l'instaurazione del regime fascista (Roma Aracne, 2010), Il confino fascista. L'arma silenziosa del regime (Roma-Bari, Laterza, 2011) e ha pubblicato saggi su numerose riviste. I suoi interessi sono focalizzati sulla storia dell'Italia e della Germania del XX secolo in prospettiva comparata e transnazionale, $\mathrm{i}$ rapporti fra fascismo italiano e nazismo, i diritti umani, l'uso della violenza, la memoria pubblica e privata, il jazz e la musica nel fascismo.

URL: < http://www.studistorici.com/progett/autori/\#Poesio > 